\title{
Perceptual and conceptual sources of priming on a word generation task
}

\author{
SHARON L. THOMPSON-SCHILL and IRENE P. KAN \\ University of Pennsylvania, Philadelphia, Pennsylvania
}

\begin{abstract}
Repetition of any number of cognitive processes can facilitate subsequent performance (i.e., repetition priming). In this study, we explored several candidate mechanisms that could account for repetition priming on a word generation task. In Experiment 1, we examined whether repetition of semantic processing is necessary for priming on this task. In Experiment 2, we examined whether repetition of semantic processing is sufficient for priming on this task. In both experiments, we additionally examined the effect of changing the specific nature of the semantic retrieval task (i.e., from visual to functional, and vice versa) on performance. The results from these experiments indicated that repetition of semantic processing is both necessary and sufficient to produce a facilitation effect on the word generation task. However, semantic processing of the same attribute does not need to be repeated for facilitation effects to occur. Implications of these findings for theories of the representation and retrieval of semantic knowledge are discussed.
\end{abstract}

Repetition priming is a well-documented facilitation effect that results from repeated exposure to a stimulus. Variations in the type and magnitude of repetition priming effects have been well explained by the theory of transfer appropriate processing (Morris, Bransford, \& Franks, 1977). According to this framework, performance on a test will benefit as a direct function of the extent to which mental processes engaged during the initial encounter with a stimulus are recapitulated during a subsequent encounter with the same stimulus. The transfer appropriate processing framework provides a logic for discovering the critical processes required while performing a task. For example, if facilitation effects are sensitive to perceptual manipulations between repeated presentations of the stimulus, but not to conceptual manipulations, one could infer that the task under investigation is driven largely by perceptual processing mechanisms. In this manner, investigations of repetition priming can be used as a means toward the goal of formulating an empirical task analysis. Many tasks used in cognitive research have been characterized in this manner, leading to a useful, if a bit too simplistic, dichotomy of perceptual (or datadriven) and conceptual (or concept-driven) tasks (Roediger, Weldon, \& Challis, 1989).

Portions of this research were presented at the 39th Annual Meeting of the Psychonomic Society, November 1998. This project was supported in part by James S. McDonnell Foundation Grant 96-29, National Institutes of Health Grants AG05743 and MH60414, and NSF Grant SES9973391. The authors thank Bradley Postle, Carol Seger, Mary Sue Weldon, Michael Masson, and two anonymous reviewers for helpful comments on an earlier version of this manuscript. The authors are also grateful for the assistance of Jessica Panzer, Farah Maloof, and Jeris Minor for their assistance in data coding. Correspondence should be addressed to S. L. Thompson-Schill, Department of Psychology, University of Pennsylvania, 3815 Walnut St., Philadelphia, PA 19104 (e-mail: sschill@psych.upenn.edu).
The focus of the present investigation was on the phenomenon of conceptual priming. In "conceptual tests," priming increases as a function of the degree of conceptual or semantic analysis required during the initial exposure to the stimulus. One example of such a test is the exemplar generation task, in which participants are cued with a category label such as fruit and are asked to generate exemplars belonging to that category (e.g., apple, pear, etc.). Uncommon target responses (e.g., mango) will be made more often following previous exposure to the target than in baseline responses; this increase in response likelihood is the facilitation effect for the exemplar generation task. Facilitation effects with this task are sensitive to manipulations of conceptual but not perceptual processes (e.g., Srinivas \& Roediger, 1990). In the present investigation, we focused on a related task, word generation, that has garnered much attention recently in light of many neuroimaging reports that have used this task to investigate the neural bases of semantic memory. In the word generation task, participants are shown a cue word (e.g., car) and are instructed to generate a new word (e.g., drive) that is related in some specified way (e.g., an action) to the cue. When the cue word is subsequently repeated, facilitation is observed as a reduction in latency to generate a response. The word generation task is similar to the exemplar generation task in many ways-most notably, in that both tasks require the production of semantically related words. However, there is at least one important difference: In the word generation task, the identical stimulus (i.e., the cue) is repeated. In contrast to perceptual tests, conceptual tests have been defined by the fact that "there is no perceptual resemblance between the priming event ... and the test cue" (Roediger \& McDermott, 1993, p. 67). The introduction of perceptual repetition into the word generation task may have implications for the nature of the resulting facilitation effect. In the 
word generation task, the cue (e.g., car), the response (e.g., drive), and the association between the cue and the response may be primed. Thus, in the word generation task, priming could be the result of repetition of any stage of processing, from the stimulus presentation to the response output.

Because there are multiple possible sources for the repetition priming effect with the word generation task, one must exercise caution in making inferences about any one cognitive process on the basis of an observed priming effect. The present study focused on one such inference that we recently made in a study of semantic processing using the word generation task (Thompson-Schill, D'Esposito, \& Kan, 1999). In the remainder of this section, we briefly review the empirical results reported in that study, the potential theoretical implications of the data, and other plausible interpretations of these findings. In the experiments presented in the present study, we have attempted to address these concerns and thereby strengthen the inferences we can draw about repetition priming effects observed with the word generation task.

Thompson-Schill, D'Esposito, and Kan (1999) reported both behavioral and physiological(i.e., fMRI) results of a priming manipulation using the word generation task. The critical manipulation in this study was the type of response that participants were instructed to generate. For each cue word, participants were instructed to generate either an action word or a color word related to the cue. On the second presentation of the cue words, the task was to generate either the same attribute (e.g., color generation followed by color generation) or a different attribute (e.g., color generation followed by action generation), relative to the first presentation. We reported a behavioral facilitation effect (i.e., faster response times) and a physiological facilitation effect (i.e., decreased blood flow in the temporal lobe measured with fMRI) for both same-attribute and different-attribute priming conditions. Similarly, a behavioral different-attribute priming effect has also been reported on a property verification task (Thompson-Schill \& Gabrieli, 1999).

Evidence for a different-attribute priming effect may have relevance for our understanding of semantic memory. Just as perceptual processing has long been accepted as a collection of modality-specific operations (i.e., auditory perceptual processing is a distinct operation from visual perceptual processing), the concept of modalityspecific operations might be extended to our understanding of conceptual processing as well. Research in several diverse areas, from language acquisition (e.g., Nelson, 1974) to language dysfunction (e.g., Warrington \& Shallice, 1984), suggests that semantic knowledge may be usefully divided into subtypes of knowledge, depending on the type of attribute that knowledge describes. Two attributes that have been extensively studied and contrasted are visual attributes (knowledge of physical properties of an object, such as color or shape) and functional attributes (knowledge of abstract properties that are not physically defined). One's knowledge of an object will typically comprise both visual and functional attributes, although there is mounting evidence that visual and functional attributes may be both behaviorally and neuroanatomically dissociable (e.g., Martin, Haxby, Lalonde, Wiggs, \& Ungerleider, 1995). Thus, it seems reasonable to consider whether processing visual semantic information and processing functional semantic information constitute discrete operations that will not result in a facilitation effect (according to the transfer appropriate processing framework) or whether there exist some common operations between the two that, when repeated, would result in a facilitation effect. Put differently, will retrieving one type of semantic knowledge about an object facilitate the subsequent retrieval of other semantic knowledge about that object?

On the basis of evidence for a different-attribute priming effect described above, we have previously argued that the answer to this question is yes (Thompson-Schill, D'Esposito, \& Kan, 1999; Thompson-Schill \& Gabrieli, 1999). Although visual semantic knowledge and functional semantic knowledge have been shown to be both behaviorally and neuroanatomically dissociable, retrieval of one type of knowledge reliably facilitated subsequent retrieval of the other type of knowledge. These demonstrations of a reliable different-attribute priming effect clearly indicate that repetition of the same attribute or domain of semantic knowledge is not necessary for priming on the word generation task. Thus, at first glance, this finding appears to have important implications for theories about the representation and organization of semantic knowledge; for example, this result would support either the claim that there exists an amodal semantic knowledge store or the claim that modality-specific knowledge stores are highly interactive. However, there are also less interesting possibilities. It is plausible, and

Table 1

Definitions and Illustrative Examples (in Italics) of the Trial Types Used in Experiments 1 and 2

\begin{tabular}{|c|c|c|}
\hline Condition & First Presentation & Second Presentation \\
\hline Unprimed & - & $\begin{array}{l}\text { generate action } \\
\text { (tomato-"slice") } \\
\text { generate color } \\
\text { (milk-"white") }\end{array}$ \\
\hline Same-attribute prime & $\begin{array}{l}\text { generate action } \\
\text { (match-"strike") } \\
\text { generate color } \\
\text { (tar-"black") }\end{array}$ & $\begin{array}{l}\text { generate action } \\
\text { ( match-"strike") } \\
\text { generate color } \\
\text { (tar-"black") }\end{array}$ \\
\hline Different-attribute prime & $\begin{array}{l}\text { generate action } \\
\text { (dollar-"spend") } \\
\text { generate color } \\
\text { (moss-"green") }\end{array}$ & $\begin{array}{l}\text { generate color } \\
\text { (dollar-"green") } \\
\text { generate action } \\
\text { (moss-"grow") }\end{array}$ \\
\hline Nonsemantic prime & $\begin{array}{l}\text { report letter } \\
\text { (apple-“p") } \\
\text { report letter } \\
(\text { knife-“ } n ")\end{array}$ & $\begin{array}{l}\text { generate color } \\
\text { (apple-"red") } \\
\text { generate action } \\
\text { (knife-"cut") }\end{array}$ \\
\hline
\end{tabular}

Note-Complete descriptions of the tasks and procedures can be found in the corresponding Method sections. 
perhaps even likely, that repetition of semantic knowledge is not in fact necessary for priming on this task. Priming on the word generation task under different-attribute conditions may have nothing to do with semantic processing at all. If this were the case, one would certainly not want to conclude anything about the representation of semantic memory on the basis of these effects.

As noted earlier, the word generation task varies from nearly all other conceptual priming tests in that the exact stimulus form is repeated at both presentations. Therefore, in addition to a repetition of semantic processes, there is also a repetition of nonsemantic processes (e.g., perceptual form analysis). In previous studies of word generation priming, there was no way to determine whether priming was the result of the repetition of semantic or nonsemantic processes. In the present study, we directly addressed this issue. In Experiment 1, we examined whether nonsemantic processing of a word will facilitate subsequent performance on the word generation task. In Experiment 2, we turned the question around, to ask whether the repetition of semantic processing, in the absence of perceptual form repetition, will produce priming on this task. Together, these two questions can help address whether the repetition of semantic processing is both necessary and sufficient to facilitate performance on the word generation task.

\section{EXPERIMENT 1}

In Experiment 1, we examined whether repetition of nonsemantic processes will facilitate performance on the word generation task, in the absence of the repetition of semantic processing. The design of this experiment was similar to that used by Thompson-Schill, D'Esposito, and Kan (1999): Participants generated related words in response to a cue that they had seen before under one of several conditions. In addition to the same-attribute and different-attribute conditions used in our earlier research, in the present experiment we added a priming condition in which there was repetition of the cue word, but with no demands for semantic processing. In this condition, the participants were asked to read the word and report the consonant in the word that was latest in the alphabet (e.g., the letter $p$ in apple). This task was selected on the basis of pilot testing that indicated that it was well matched in difficulty (e.g., equivalent latencies) to the action and color generation tasks. An examination of the facilitation effect that results from this nonsemantic priming task enabled us to address the question, Is repetition of semantic processing necessary for priming on the word generation task?

\section{Method}

Participants. Forty-eight undergraduate and graduate students from the University of Pennsylvania took part in the experiment and were paid $\$ 7$ each for their participation. The participants were tested individually. Informed consent was obtained from all participants after the nature and possible consequences of the procedure were explained.

Materials. A list of 288 concrete nouns (e.g., chair) was selected as stimuli for the cue words. Median word length for the list was 5, the Kučera-Francis (Kučera \& Francis, 1967) frequency was 9 , and the median imageability rating (Coltheart, 1981) for the concrete nouns was 595 . The experiment was divided into two phases (i.e., a study phase and a test phase). There were 192 trials in the study phase, grouped into blocks of 8 trials of the same task.

During each block of trials, the participants performed one of three tasks: For 48 trials, the participants saw a novel concrete noun and generated a related action word for each cue word. For 48 trials, the participants saw a novel concrete noun and generated a related color word for each cue word. For 96 trials, the participants saw a novel concrete noun and reported the letter in the word that was latest in the alphabet. On the basis of pilot testing, the instructions were modified to require the consonant that was latest in the alphabet; this change was made due to a very high number of errors in which the participants neglected the vowels before we instructed them to do so. So, for example, the correct response would be " $g$ " for fudge and " $t$ " for tongue. The presentation order of the trial blocks was pseudorandomized, such that the participants would never encounter the same type of trials in two consecutive sets, and the order was counterbalanced across subjects.

The test phase was composed of 288 trials. Stimuli in the test phase comprised both repeated items (i.e., all 192 words from the study phase) and novel items (i.e., 96 novel concrete nouns). In the test phase, the participants performed only the action and color generation tasks. As with the study phase, these trials were arranged into blocks of 8 trials according to trial type. For 96 trials, the participants saw a novel concrete noun and generated either a related action word (48 trials) or a related color word (48 trials) for each cue word. For 192 trials, the participants saw a repeated concrete noun and generated either a related action word (96 trials) or a related color word (96 trials) for each cue word: For half of these trials, the previous presentation of the word was in the nonsemantic letter condition, and, for the other half of the trials, the previous presentation of the word was in one of the semantic conditions (either color or action), divided evenly between same-attribute prime trials and different-attribute prime trials. The conditions were counterbalanced between subjects, so, across 16 counterbalancing orders, each concrete noun appeared in each of the four priming conditions (sameattribute, different-attribute, nonsemantic, and unprimed) and in each of the two task conditions (action and color). Examples of these types of trials are provided in Table 1 .

Procedure. Stimuli were presented in lowercase letters on a Power Macintosh 6500 using PsyScope experimental software (Carnegie Mellon University, Pittsburgh, PA) to display the stimuli. A PsyScope button box with a voice-activated relay was utilized to record the latency until onset of a verbal response for each trial. Responses were also recorded on audio tapes for subsequent evaluation. Any trial on which there was an error with the voice-activated relay was noted and ultimately eliminated from subsequent response analyses (fewer than $2 \%$ of trials); these trials do not affect the response times or the error rates reported here.

Each block of 8 trials began with a 400 -msec task prompt (i.e., LETTER, ACTION, or COLOR during the study phase, and ACTION or COLOR during the test phase), which was presented in uppercase letters in the middle of the computer screen. The task prompt remained on the screen for the duration of the block. At the beginning of each trial, the stimulus cue word appeared inside a rectangular box below the task prompt. The cue remained on the screen until the participants made their response verbally. A 400-msec intertrial interval then followed. The participants were encouraged to respond as quickly as possible without making mistakes. 
The participants were given instructions and examples of each of the three tasks (letter, action, and color). For the letter trials, the participants were told to report the consonant in the word that was latest in the alphabet. For the action trials, the participants were told to say aloud an action word that was either something the object did or something you could do with the object. For the color trials, the participants were told to say aloud a color word that described what color the object typically was. For action and color trials, the participants were instructed to say the first appropriate word that came to mind, even if it was a response they had already given for a different cue word. The participants were given no instructions about the repetition of stimuli throughout the experiment. Prior to beginning the experimental trials, the participants were given 54 practice trials, to familiarize them with the experimental paradigm and the task instructions and to allow the experimenter to adjust the sensitivity of the voice-activated relay. If there were no remaining questions, the practice trials were immediately followed by the study phase, which consisted of 192 trials. After a brief unfilled break, the test phase began, and 288 trials were presented. The lag between the initial presentation and repeated presentation of each item was approximately $8 \mathrm{~min}$.

\section{Results}

For each type of trial, median response times were calculated for each participant, after discarding trials with voice-activated relay problems or participant errors (see Table 2). These median response times were then analyzed in a within-subjects analysis of variance (ANOVA) of trial type (unprimed, same-attribute, different-attribute, nonsemantic). In the initial analysis, we included attribute type as a within-subjects factor (color response or action response), so we could examine any differences in priming for these conditions. Although there was a main effect of attribute [color responses were generated more quickly than action responses; $F(1,141)=138.09, p<$ $.01]$, this effect did not interact with trial type $(F<1)$. For this reason, and in the interest of simplicity, we have collapsed across attribute type for the remainder of the analyses and in Table 2.

The one-way ANOVA revealed a main effect of trial type $[F(3,141)=32.79, p<.01]$. To examine pairwise differences between conditions, paired $t$ tests were calculated between trial types. Response latencies were longer in the unprimed condition than in both the differentattribute priming condition $[t(47)=2.23, p<.05]$ and the same-attribute priming condition $[t(47)=9.47, p<$
.01]. Additionally, response latencies were longer in the different-attribute priming condition than in the sameattribute priming condition $[t(47)=4.81, p<.01]$. When these two semantic priming conditions were compared with the nonsemantic priming condition, we found that response latencies were longer in the nonsemantic condition than in the semantic conditions $[t(47)=7.55, p<$ .01]. Of central importance for this experiment, there was no difference between response latencies in the nonsemantic condition and in the unprimed condition $[t(47)=0.78]$. To ensure that there was sufficient power to obtain a facilitation effect, we calculated our power to detect an effect similar in magnitude to the differentattribute priming effect we observed in Thompson-Schill, D'Esposito, and Kan (1999; $46 \mathrm{msec})$; with our sample size and estimated variability of the effect size, power > .80. Because some of the preceding comparisons involved conditions with unequal numbers of trials, we repeated the preceding analyses using trimmed means instead of median latencies (Van Selst \& Jolicœur, 1994). There were no changes to any of the results with this analysis. Notably, there was a priming effect for the semantic conditions $(p<.01)$, but not for the nonsemantic condition $(t<1)$.

After excluding voice-activated relay mistrials, error rates were calculated for each type of trial. Two types of errors occurred: (1) The participant read the cue word instead of generating a response, and (2) the participant generated the wrong type of response (e.g., an action word during a color trial). Accuracy rates approached ceiling levels of performance for most participants (see Table 2). Perhaps, therefore, not surprisingly, an ANOVA indicated no effect of trial type on accuracy $[F(3,141)=$ $2.39]$. No pairwise $t$ tests for any condition versus the unprimed condition were significant (all $p \mathrm{~s}>.15$ ).

To verify that the semantic and nonsemantic tasks used in the study phase were adequately matched in response time, in order to ensure that the perceptual exposure to the stimulus was comparable across conditions, we also analyzed the response latencies from the study phase. Response times for the letter task $(M=1,280 \mathrm{msec})$ were comparable to latencies for both the action task $(M=$ $1,437 \mathrm{msec})$ and the color task $(M=1,207 \mathrm{msec})$. A $t$ test

Table 2

Response Latency and Accuracy for Unprimed, Same-Attribute Semantic Primed, Different-Attribute Semantic Primed, and Nonsemantic Primed Items $(N=48)$ in Experiment 1

\begin{tabular}{|c|c|c|c|c|c|}
\hline \multirow[b]{2}{*}{ Condition } & \multicolumn{2}{|c|}{ Latency (in msec) } & \multicolumn{2}{|c|}{$\mathrm{PE}$} & \multirow{2}{*}{$\begin{array}{c}\text { Mean } \\
\text { Accuracy (in \%) }\end{array}$} \\
\hline & $M$ & $S E$ & $M$ & $S E$ & \\
\hline Unprimed & $1,378.83$ & 54.90 & - & - & 98 \\
\hline Semantic prime & $1,240.14$ & 46.34 & 139.70 & 18.37 & 97 \\
\hline Same-attribute & $1,160.23$ & 45.77 & 218.60 & 23.07 & 98 \\
\hline Different-attribute & $1,320.04$ & 52.50 & 58.79 & 26.36 & 97 \\
\hline Nonsemantic prime & $1,365.56$ & 51.22 & 13.27 & 16.91 & 98 \\
\hline
\end{tabular}

Note-PE refers to priming effect on response latency (unprimed - primed); accuracy $S E<1 \%$ in all conditions. 
comparing latencies from the letter task with the average of the two semantic tasks revealed no differences between conditions in the study phase $[t(47)=1.01]$.

\section{Discussion}

In this experiment, we examined the facilitation effects on word generation latency of the identical stimulus cue processed under one of three conditions. In the same-attribute and different-attribute conditions, semantic processing of the cue word primed subsequent semantic processing on the word generation task. In the nonsemantic condition, processing of the cue word did not prime subsequent semantic processing on the word generation task. The pattern of results with the same-attribute and different-attribute conditions replicates our previous findings (Thompson-Schill, D’Esposito, \& Kan, 1999), despite several methodological changes; in fact, although these changes may have affected the absolute response time, the relative magnitude of the facilitation effect was remarkably similar in the two experiments. The percent savings in response time for the same-attribute prime condition was $15.3 \%$ in Thompson-Schill, D'Esposito, and Kan and $15.9 \%$ in this experiment; for the differentattribute prime condition, it was $4.4 \%$ in ThompsonSchill, D'Esposito, and Kan and $4.3 \%$ in this experiment. Thus, our conclusions appear to be robust to at least minor procedural variations.

The nonsemantic priming condition was included in this experiment in order to address whether repetition of semantic processing was necessary to produce a reliable facilitation effect. This condition was similar to the semantic priming conditions in that it had the identical perceptual and lexical form. To ensure that any diminution in priming effects was not the result of decreased perceptual exposure, we used a task that was matched in terms of response latency with the semantic tasks; this was verified by the absence of any differences in latencies in the study phase across conditions. Thus, any differences in facilitation effects between the semantic and nonsemantic tasks would most likely be attributable to the absence of semantic processing that occurred during the letter task. Indeed, the nonsemantic priming condition did not produce reliable facilitation, despite adequate power to detect this effect. There are a few caveats about this choice of task. Although we have suggested that the letter task required perceptual and lexical processing, we actually know very little about the task demands of this condition. On the one hand, it is possible that there is some semantic processing in our putative nonsemantic task; conditions that on the surface have few demands for meaningful processing might still result in some processing of semantic information. Despite this possibility, we did not observe priming in this condition. On the other hand, it is possible that it is a purely perceptual task and does not require semantic or lexical information. If this were the case, semantic and lexical processing would be confounded in our conditions. Acknowledging these caveats about the task, we can still safely conclude that repetition of semantic or lexical processing is in fact necessary to produce a facilitation effect on the word generation task, although semantic processing of the same attribute is not required.

\section{EXPERIMENT 2}

Facilitation on the word generation task appears to depend critically on repetition of semantic processing. Furthermore, it is clear from the preceding experiment that this semantic repetition need not be task-specific (i.e., same-attribute). These experiments inform us as to the necessity of semantic processing for priming on the word generation task. In Experiment 2, we examined whether semantic processing is sufficient to produce priming on this task by decreasing the potential contribution of perceptual sources of facilitation. In many prior studies, a change in stimulus modality between the two presentations of the stimulus eliminated or substantially reduced the facilitation effect on perceptual tests (e.g., Jacoby \& Dallas, 1981) but not on conceptual tests (e.g., Srinivas \& Roediger, 1990). These studies indicate that modality change is an effective way to decrease perceptual influences on priming.

In Experiment 2, we manipulated the modality of the cue word between the two presentations of the stimulus (same-modality or different-modality) and examined facilitation on the word generation task in both the sameattribute prime condition and the different-attribute prime condition. This design allowed us to manipulate a number of processes that were plausible candidates for the facilitation effects we have observed. In the same-attribute same-modality condition, there was repetition of the perceptual form of the stimulus, the attribute-specific semantic processing, and the response (the response was repeated by the participant on most trials; see the General Discussion section for more detail) and the association between the stimulus and the response. In the sameattribute different-modality condition, there was repetition of all of the above except perceptual form. In the differentattribute same-modality condition, there was repetition of the perceptual form and of nonspecific semantic processing. Critically, in the different-attribute differentmodality condition, only nonspecific semantic processing was repeated; the perceptual form, the semantic attribute, and the response all varied between presentations. Thus, any facilitation we observed in the different-attribute different-modality condition was most likely the result of the repetition of nonspecific semantic processing.

\section{Method}

Participants. Forty-eight undergraduate and graduate students from the University of Pennsylvania took part in the experiment and were paid $\$ 7$ each for their participation. The participants were tested individually. Informed consent was obtained from all participants after the nature and possible consequences of the procedure were explained. 
Materials. The stimuli used in this experiment were the same 288 concrete nouns used in Experiment 1. For auditory presentations of the stimuli, words were recorded by a male native English speaker and were played via the computer speakers. The duration of each auditory presentation ranged from 301 to $1,091 \mathrm{msec}$, with a median of $650 \mathrm{msec}$. Duration of visual presentation was matched to the median of the duration of presentation for auditory stimuli.

The experiment was divided into two phases (i.e., a study phase and a test phase). A total of 192 trials were included in the study phase. On each trial, the participants performed one of four tasks: For 48 trials, the participants heard a novel concrete noun and generated a related action word for each cue word. For 48 trials, the participants heard a novel concrete noun and generated a related color word for each cue word. For 48 trials, the participants saw a novel concrete noun and generated a related action word for each cue word. For 48 trials, the participants saw a novel concrete noun and generated a related color word for each cue word. Unlike the previous experiment, trial types were not blocked in this experiment; the presentation order was pseudorandomized, such that no more than 4 consecutive trials were drawn from the same trial type.

The test phase was composed of 288 trials. Stimuli in the test phase comprised both repeated items (i.e., all 192 words from the study phase) and novel items (i.e., 96 novel concrete nouns). In the test phase, all items were presented visually. For 96 trials, the participants saw a novel concrete noun and generated either a related action word (48 trials) or a related color word (48 trials) for each cue word. For 192 trials, the participants saw a repeated concrete noun and generated either a related action word ( 96 trials) or a related color word ( 96 trials) for each cue word. For half of these trials, the participants generated the same type of response as during the initial presentation (same-attribute prime condition); for the other half of these trials, the participants generated a different type of response (different-attribute prime condition). In each of these conditions, half of the trials were items that were presented auditorily in the study phase (different-modality condition), and half of the trials were items that were presented visually in the study phase (same-modality condition). These two factors (study task and study modality) were fully crossed, with 48 trials of each of the resulting four conditions in the test phase (collapsing over test task). As with the study phase, the presentation order was pseudorandomized. The conditions were counterbalanced between subjects, so, across 12 counterbalancing orders, each concrete noun appeared in each of the three priming conditions (same-attribute, different-attribute, or unprimed), in each of the two task conditions (action or color), and in each of the two modality conditions (same-modality or different-modality).

Procedure. The general procedure was identical to that of Experiment 1, with the following two exceptions: First, as noted above, the trial presentation order was randomized in this experiment instead of blocked. Therefore, in order to instruct the participant which task to perform, a task prompt was presented on every trial $600 \mathrm{msec}$ prior to the cue word presentation. Second, during the study phase only, an additional prompt was presented to alert the participants as to whether the stimulus would be visual or auditory. Thus, in the study phase, each trial began with a modality prompt (LOOK or LISTEN) for $600 \mathrm{msec}$, followed by a task prompt (ACTION or COLOR) for $600 \mathrm{msec}$. The task prompt remained on the screen, while the stimulus cue word was presented either visually, inside a rectangular box below the task prompt (exactly as before), or auditorily, via computer speakers. (For the auditory trials, the modality and task prompts and the box were still presented visually.) The presentation of the visual stimuli was adjusted to resemble the auditory presentation as closely as possible; therefore, each visual stimulus remained on the screen for only $650 \mathrm{msec}$, which was the median length of the duration of auditory presentation. (This stimulus duration was also used in the test phase.) Both the task prompt and the rectangular box remained on the screen until the participants made their response verbally. As with Experiment 1, the participants had unlimited time to make their response.

\section{Results}

For each type of trial, median response times were calculated for each participant, after discarding trials with voice-activated relay problems or participant errors (see Table 3 ). In addition, trials were excluded in which the participant did not correctly hear or understand the auditory presentation of the item in the study phase (fewer than $1.5 \%$ of trials).

As in Experiment 1, we initially conducted an ANOVA with attribute type (action or color) as a within-subjects factor. Although there was a main effect of attribute $[F(1,47)=56.97, p<.01]$, this factor did not interact with any other factor in the analysis, and all subsequent analyses reported here have collapsed the data across both attribute types.

A two-way ANOVA of trial type (unprimed, sameattribute, different-attribute) by modality (same-modality, different-modality) revealed a main effect of trial type $[F(2,47)=57.98, p<.01]$. There was no effect of modality $[F(1,47)=3.89]$ and, critically, no interaction between modality and prime type $[F(2,47)=0.13]$. To ensure that there was sufficient power to obtain a modality effect, we calculated our power to detect an effect similar in magnitude to the different-attribute priming effect we observed in Thompson-Schill, D'Esposito, and Kan

Table 3

Response Latency and Accuracy for Unprimed, Same-Attribute Primed, and Different-Attribute Primed Items Presented in Either the Same or a Different Modality $(N=48)$ in Experiment 2

\begin{tabular}{|c|c|c|c|c|c|}
\hline \multirow[b]{2}{*}{ Condition } & \multicolumn{2}{|c|}{ Latency (in msec) } & \multicolumn{2}{|c|}{$\mathrm{PE}$} & \multirow{2}{*}{$\begin{array}{c}\text { Mean } \\
\text { Accuracy (in \%) }\end{array}$} \\
\hline & $M$ & $S E$ & $M$ & $S E$ & \\
\hline Unprimed & $1,526.06$ & 62.72 & - & - & 99 \\
\hline \multicolumn{6}{|l|}{ Same-attribute prime } \\
\hline Same-modality & $1,304.50$ & 48.34 & 221.56 & 29.92 & 99 \\
\hline Different-modality & $1,329.98$ & 48.85 & 196.08 & 23.91 & 99 \\
\hline \multicolumn{6}{|c|}{ Different-attribute prime } \\
\hline Same-modality & $1,461.63$ & 57.14 & 64.44 & 16.69 & 98 \\
\hline Different-modality & $1,475.96$ & 57.74 & 50.10 & 20.14 & 98 \\
\hline
\end{tabular}

Note-PE refers to priming effect on response latency (unprimed - primed); accuracy

$S E<1 \%$ in all conditions. 
(1999; 46 msec); with our sample size and estimated variability of the effect size, power $>.90$. (We also calculated a post hoc analysis of the power to detect an effect that was a $40 \%$ reduction in the different-attribute priming effect we observed [39 $\mathrm{msec}$ ], which is comparable to the modality effects observed in perceptual priming experiments; with our sample size and estimated variability of the effect size, power $>.80$.)

To test for a facilitation effect in each of the four conditions, pairwise differences between each condition against the unprimed condition were calculated. To maintain the overall $\alpha=.05$, a Bonferroni-corrected significance level for one-tailed tests of .025 was used. For sameattribute primes, response latencies were longer in the unprimed condition than in the primed conditions for both the same-modality condition $[t(47)=7.40, p<.025]$ and the different-modality condition $[t(47)=8.20, p<.025]$. Likewise, for different-attribute primes, response latencies were longer in the unprimed condition than in the primed conditions for both the same-modality condition $[t(47)=3.86, p<.025]$ and the different-modality condition $[t(47)=2.49, p<.025]$. When data were collapsed across the modality factor, the effects reported in Experiment 1 were replicated: Response latencies were longer in the unprimed condition than in both the different-attribute priming condition $[t(47)=3.82, p<.01]$ and the sameattribute priming condition $[t(47)=8.31, p<.01]$. Additionally, response latencies were longer in the differentattribute priming condition than in the same-attribute priming condition $[t(47)=7.75, p<.01]$. These analyses were repeated using trimmed means instead of medians with no change to the findings reported above. The critical interaction between modality and trial type did not approach significance $(F<1)$. As with Experiment 1 , accuracy rates were at ceiling levels of performance for most participants (see Table 3 ). In pairwise comparisons of each priming condition against the unprimed condition, only one comparison was significant: Accuracy was significantly lower in the same-modality different-attribute prime condition than in the unprimed condition $[t(47)=$ $2.74, p<.05]$. No other comparisons were significant.

\section{Discussion}

As in Experiment 1, we observed a reliable facilitation effect on the word generation task in both the sameattribute condition and the different-attribute condition. Critically for this experiment, there was no effect of changing modality between the two presentations of the stimulus; facilitation in the different-attribute prime condition persisted in the absence of repetition of the stimulus form, indicating that repetition of nonspecific semantic processing is sufficient for priming on the word generation task.

\section{GENERAL DISCUSSION}

In this study, we examined whether repetition of semantic processing is both necessary and sufficient to facili- tate performance on a word generation task. Across both experiments, two effects were reliably and consistently observed. First, priming of different-attribute semantic information produced a $4 \%$ reduction from the baseline (unprimed) response time, just as in our previous demonstration of this effect (Thompson-Schill, D'Esposito, \& Kan, 1999), despite changes in stimulus duration and modality, trial randomization, response time limit, and lag between the first and second presentations. Second, priming of same-attribute semantic information produced an enhancement in this facilitation effect of approximately $15 \%$, again despite numerous methodological changes. This effect size is also comparable to that reported in other studies of word generation priming (e.g., Seger, Rabin, Desmond, \& Gabrieli, 1999).

There are two general issues for which these findings have relevance. First, what is the basis for priming on the word generation task? As noted in the introduction, several methodological differences between this task and other conceptual tests raise a number of possibilities that have not existed in previous studies. Second, what can these priming effects tell us about the organization and retrieval of semantic memory? The subtypes of semantic knowledge that were manipulated in this series of experiments (i.e., visual and functional) were chosen because they appear to have both behavioral and neuropsychological relevance. These two questions will be discussed in turn.

At the outset of this study, we considered a number of possible mechanisms that could potentially produce facilitation on the word generation task, and our findings enable us to confidently rule out several of these. First, we are confident that repetition of perceptual processing is not necessary for priming on this task. It is possible that perceptual repetition might produce a slight enhancement of the facilitation effect; although we did not detect such an effect in our present study despite adequate power, it may be noteworthy that the smallest priming effects across the experiments were found in the differentmodality conditions. Second, we found evidence that repetition of response-specific processing is not necessary for priming on this task. This possibility was raised by Seger et al. (1999), who argued that the source of the priming effect in the word generation task is responsespecific and independent of the identity of the cue word; yet we observed priming across two presentations in which there was no repetition of the response. Alternatively, we suggest that facilitation on the word generation task requires the repetition of semantic processing of any aspect of the stimulus-response association. Our experiments show that priming the stimulus concept is sufficient to produce facilitation, whereas Seger et al. show that priming the response concept is sufficient to produce facilitation. We have also shown that priming both the stimulus and the response (Experiment 1) produces a greatly enhanced facilitation effect. Third, we found evidence that repetition of processing of the same attribute or domain of semantic knowledge is not necessary 
for priming on this task. This finding may have implications for the theories of the representation and retrieval of semantic knowledge.

Psychologists and neuropsychologists have long been interested in uncovering the organizational structure of semantic knowledge. Early models of semantic networks were organized categorically and hierarchically (e.g., Collins \& Quillian, 1969; Quillian, 1966). Alternatively, Allport (1985) hypothesized that semantic memory is subdivided into different sensorimotor modalities (e.g., visual knowledge); he suggested that there was not a separate semantic memory store but rather that semantic knowledge was embodied in representations within each sensorimotor system. Both of these alternatives - categoryspecific representations and modality-specific representations-have been to some extent bolstered by neuropsychological evidence for category-specific knowledge deficits (e.g., Warrington \& Shallice, 1984). Although this issue continues to be debated, there is mounting evidence for the existence of neuroanatomically distinct modality-specific knowledge stores (Martin et al., 1995).

A related question is whether retrieval of knowledge from different stores proceed independently, or whether there is interaction between these systems. The computation model of Farah and McClelland (1991) is based on a semantic memory network that is organized into distinct but highly interactive sensorimotor stores. This interactivity was confirmed in a recent neuroimaging study, which found that retrieval of one type of knowledge could depend on retrieval of another type of knowledge in certain circumstances; for example, retrieving nonvisual knowledge about an entity that is described mostly by visual information, such as an animal, required the additional retrieval of visual knowledge (Thompson-Schill, Aguirre, D'Esposito, \& Farah, 1999). The notion that retrieval from any one semantic knowledge representation might require interactive support from other knowledge representations is inconsistent with the idea that there are sensorimotor semantic modules that can be independently accessed.

Although the present study was not specifically designed to resolve the issue of the independence of sensorimotor semantic memory stores, we chose to query the participants about visual and functional domains of semantic knowledge in order to examine some of the questions raised above. Our evidence for a reliable facilitation effect from different-attribute semantic primes is consistent with two alternatives related to the representation of semantic knowledge. First, semantic memory may comprise both modality-specific semantic memory stores and nonspecific stores, which could resemble modality-free semantic networks, after Quillian (1966), or any other type of associative network; facilitation from different-attribute primes could result from the recapitulation of processing of these nonspecific semantic memory representations. Second, all semantic memory representations may be modality-specific, but the interactions between these stores, described above, would lead to some priming from the retrieval of irrelevant semantic knowledge. That is, when we found in our experiments, for example, that retrieving functional information primed subsequent retrieval of visual information, it could be that the retrieval of functional information elicited, as a result of the interactivity between these stores, some degree of retrieval of visual information, and it was this visual retrieval that primed the subsequent visual retrieval attempt. Although our results do not distinguish between these two interesting alternatives, this priming technique could be used in the future to address this and other questions about the organization of semantic memory.

There is another, less interesting explanation for the different-attribute prime effect: As a consequence of the within-subjects manipulation that required the participants to retrieve either functional or visual information on a given trial, the participants could have developed a strategy of retrieving both functional and visual information on every trial. There are three reasons to think this is not the case. First, the effect was the same when the procedure required a speeded response (ThompsonSchill, D'Esposito, \& Kan, 1999) as when there was no response time limit (Experiments 1 and 2); one might predict that, in a speeded response situation, retrieving unnecessary information would not be an effective strategy. Second, the effect was the same when the materials were blocked according to task (Experiment 1) as when they were randomly intermixed (Experiment 2); one might predict that a mixed presentation order would be more likely to promote use of such a strategy. Third, in Experiment 1 , there was no facilitation in the nonsemantic prime condition; therefore, this explanation would need to account for why the strategy would be used on some trials but not on others.

Thus far, we have focused exclusively on the implications of the different-attribute priming effect. Perhaps equally important is the consistent enhancement of the facilitation effect in the same-attribute priming condition relative to different-attribute primes. There are several possible explanations of this effect. First, this enhancement could reflect the repetition of perceptual processing in the same-attribute prime condition; however, since this enhancement persists in the absence of perceptual repetition (Experiment 2), this cannot be the sole factor. Second, this enhancement could reflect the repetition of the response and of the stimulus-response association in the same-attribute prime condition. In previous research, using a semantic classification task, we demonstrated a similar enhancement even in a same-attribute prime condition in which there was no repetition of the response, although the enhancement was somewhat attenuated (Thompson-Schill \& Gabrieli, 1999). Thus, response repetition seems likely to be just one contributing factor in this effect. Third, this enhancement could reflect the repetition of modality-specific semantic retrieval processes. Fourth, this enhancement could reflect the facilitation of nonsemantic processes. In related work, we have discussed selection processes involved in word generation tasks (Thompson-Schill, D’Esposito, Aguirre, \& 
Farah, 1997; Thompson-Schill et al., 1998) that would be facilitated in the same-attribute priming condition but not in the different-attribute condition (ThompsonSchill, D’Esposito, \& Kan, 1999). We predict that most or all of these factors have some role in the effects we have observed, and future research will be necessary to understand each in more detail.

\section{REFERENCES}

Allport, D. A. (1985). Distributed memory, modular subsystems and dysphasia. In S. K. Newman \& R. Epstein (Eds.), Current perspectives in dysphasia (pp. 207-244). Edinburgh: Churchill Livingstone.

Collins, A. M., \& Quillian, M. R (1969). Retrieval time from semantic memory. Journal of Verbal Learning \& Verbal Behavior, 8, 240-248.

Coltheart, M. (1981). The MRC psycholinguistic database. Quarterly Journal of Experimental Psychology, 33A, 497-505.

FARAH, M. J., \& McClelland, J. L. (1991). A computational model of semantic memory impairment: Modality specificity and emergent category specificity. Journal of Experimental Psychology: General, 120, 339-357.

JACOBY, L. L., \& Dallas, M. (1981). On the relationship between autobiographical memory and perceptual learning. Journal of Experimental Psychology: General, 110, 306-340.

KuČERA, H., \& FrANCIS, W. N. (1967). Computationalanalysis of presentday American English. Providence, RI: Brown University Press.

Martin, A., Haxby, J. V., Lalonde, F. M., Wiggs, C. L., \& UngerLEIDER, L. G. (1995). Discrete cortical regions associated with knowledge of color and knowledge of action. Science, 270, 102-105.

Morris, C. D., Bransford, J. D., \& Franks, J. J. (1977). Levels of processing versus transfer appropriate processing. Journal of Verbal Learning \& Verbal Behavior, 16, 519-533.

Nelson, K. (1974). Concept, word, and sentence: Interrelations in acquisition and development. Psychological Review, 81, 267-285.

Quillian, M. R. (1966). Semantic memory. Cambridge, MA: Bolt, Beranak \& Newman.
Roediger, H. L., III, \& McDermott, K. B. (1993). Implicit memory in normal human subjects. In F. Boller \& J. Grafman (Eds.), Handbook of neuropsychology (pp. 63-131). Amsterdam: Elsevier.

Roediger, H. L., III, Weldon, M. S., \& Challis, N. H. (1989). Explaining dissociations between implicit and explicit measures of retention: A processing account. In H. L. Roediger III \& F. I. M. Craik (Eds.), Varieties of memory and consciousness: Essays in honour of Endel Tulving (pp. 3-14). Hillsdale, NJ: Erlbaum.

Seger, C. A., Rabin, L. A., Desmond, J. E., \& Gabrieli, J. D. E. (1999). Verb generation priming involves conceptual implicit memory. Brain \& Cognition, 41, 150-177.

Srinivas, K., \& Roediger, H. L., III (1990). Classifying implicit memory tests: Category association and anagram solution. Journal of Memory \& Language, 29, 389-412.

Thompson-Schill, S. L., Aguirre, G. K., D’Esposito, M., \& Farah, M. J. (1999). A neural basis for category and modality specificity of semantic knowledge. Neuropsychologia, 37, 671-676.

Thompson-Schill, S. L., D'Esposito, M., Aguirre, G. K., \& Farah, M. J. (1997). Role of left inferior prefrontal cortex in retrieval of semantic knowledge: A reevaluation. Proceedings of the NationalAcademy of Sciences, 94, 14792-14797.

Thompson-Schill, S. L., D'Esposito, M., \& Kan, I. P. (1999). Effects of repetition and competition on prefrontal activity during word generation. Neuron, 23, 513-522.

Thompson-Schill, S. L., \& Gabrieli, J. D. E. (1999). Priming of visual and function knowledge on a semantic classification task. Journal of Experimental Psychology: Learning, Memory, \& Cognition, 25, 41-53.

Thompson-Schill, S. L., Swick, D., Farah, M. J., D'Esposito, M., KAN, I. P., \& KNIGHT, R. T. (1998). Verb generation in patients with focal frontal lesions: A neuropsychological test of neuroimaging findings. Proceedings of the National Academy of Sciences, 96, 1585515860.

VAN SElST, M., \& Jolicceur, P. (1994). A solution to the effect of sample size on outlier elimination. Quarterly Journal of Experimental Psychology, 47A, 631-650.

Warrington, E. K., \& Shallice, T. (1984). Category specific semantic impairments. Brain, 107, 829-854.

(Manuscript received January 27, 1999;

revision accepted for publication January 21,2001.) 